\title{
THE CHARACTERIZATION OF THE ALMOST PERIODIC ULTRADISTRIBUTIONS OF BEURLING TYPE
}

\author{
IOANA CIORANESCU
}

(Communicated by Palle E. T. Jorgensen)

\begin{abstract}
We introduce and study the space of almost periodic ultradistributions of Beurling type and characterize it in terms of classical Bohr almost periodicity. To this aim we establish a structure theorem for the bounded ultradistributions.

Application is made to the Dirichlet Problem for the half plane with almost periodic untradistributional boundary values.
\end{abstract}

\section{INTRODUCTION}

First we introduce the spaces of functions and ultradistributions that are called to play a basic role in our considerations. The notations and definitions are to be found in $[4,7]$.

Let $\left(M_{p}\right), p \in N_{0}=N \cup\{0\}$, be a sequence of positive numbers with the properties:

(M.1) logarithmic convexity:

$$
M_{p}^{2} \leq M_{p-1} M_{p+1}, \quad p \in N ;
$$

(M.2) stability under ultradifferential operators:

$$
M_{p} \leq A H^{p} \min _{q} M_{q} M_{p-q}, \quad p \in N_{0},
$$

for some positive constants $A$ and $H$;

(M.3) strong non-quasi-analyticity:

$$
\sum_{q=p+1}^{\infty} M_{q-1} / M_{q} \leq A p M_{p} / M_{p+1}, \quad p \in N,
$$

for some positive constant $A$. An example of such a sequence is the Gervey sequence $M_{p}=p !^{d}, d>1$.

We define the associated function $M$ on $(0, \infty)$ by

$$
M(t)=\sup \log _{p \geq 0} M_{0} t^{p} / M_{p} .
$$

Received by the editors February 13, 1991.

1991 Mathematics Subject Classification. Primary 46F05. 
For $h>0$ and $K \subset R$ a compact set, we denote

$$
\begin{aligned}
& \mathbf{E}\left(\left(M_{p}\right), K, h\right)=\left\{f \in C^{\infty}(R) ;\|f\|_{K, h}=\sup _{t \in K, p \in N_{0}}\left|f^{(p)}(t)\right| / h^{p} M_{p}<\infty\right\}, \\
& \mathbf{D}\left(\left(M_{p}\right), K, h\right)=\left\{\varphi \in \mathbf{E}\left(\left(M_{p}\right), K, h\right) ; \operatorname{supp} \varphi \subset K\right\} .
\end{aligned}
$$

Then $\mathbf{E}\left(\left(M_{p}\right), K, h\right)$ and $\mathbf{D}\left(\left(M_{p}\right), K, h\right)$ are Banach spaces under the norm $\|\cdot\|_{K, h}$. For a compact exhaustion $\left(K_{n}\right) ; n \in N$, of $R$ we define the following spaces of ultradifferentiable functions of class $\left(M_{p}\right)$, of Beurling type

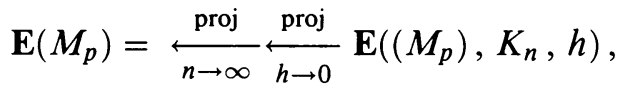

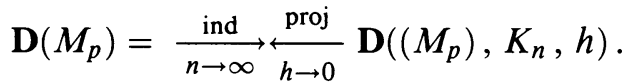

Then the strong dual $\mathbf{D}^{\prime}\left(M_{p}\right)$ is the space of (Beurling) ultradistributions of class $\left(M_{p}\right)$ and $\mathbf{E}^{\prime}\left(M_{p}\right)$ is the subspace of ultradistributions of class $\left(M_{p}\right)$ with compact support on $R$.

Further we denote

$$
\begin{aligned}
& \mathbf{D}_{L^{1}}=\left\{f \in C^{\infty}(R) ;\left\|f^{(p)}\right\|_{1}=\int_{-\infty}^{+\infty}\left|f^{(p)}(t)\right| d t<\infty, p \in N_{0}\right\}, \\
& \mathbf{D}_{L^{1}}\left(\left(M_{p}\right), h\right)=\left\{f \in \mathbf{D}_{L^{1}} ;\|f\|_{1, h}=\sup _{p \in N_{0}}\left\|f^{(p)}\right\|_{1} / h^{p} M_{p}<\infty\right\},
\end{aligned}
$$

and

$$
\mathbf{D}_{L^{1}}\left(M_{p}\right)=\stackrel{\text { proj }}{\stackrel{h \rightarrow 0}{D^{\prime}}} \mathbf{D}_{L^{1}}\left(\left(M_{p}\right), h\right) \text {. }
$$

Then $\mathbf{D}_{L^{1}}\left(\left(M_{p}\right), h\right)$ is Banach space, $\mathbf{D}\left(M_{p}\right)$ is dense in $\mathbf{D}_{L^{1}}\left(M_{p}\right)$, and $\mathbf{D}_{L^{1}}\left(M_{p}\right)$ is dense in $\mathbf{D}_{L^{1}}$. The ultradistributions of class $\left(M_{p}\right)$ that are in the strong dual $\mathbf{D}_{L^{1}}^{\prime}\left(M_{p}\right)$ are called bounded ultradistributions of class $\left(M_{p}\right)$. Finally we recall the following notations:

$$
\begin{aligned}
C_{b} & =\left\{f \in C^{0}(R) ; \sup _{t \in R}|f(t)|<\infty\right\}, \\
C_{\text {ap }} & =\left\{f \in C_{b} ; f \text { is (Bohr) almost periodic on } R\right\} .
\end{aligned}
$$

Definition. $T \in \mathbf{D}^{\prime}\left(M_{p}\right)$ is called almost periodic, in short a.p., if $T$ is bounded and is the limit of a sequence of trigonometric polynomials in the space $\mathbf{D}_{L^{1}}^{\prime}\left(M_{p}\right)$.

We recall that Schwartz [8] studied the class of the almost periodic distributions. Petzsche [6] and more recently Taguchi [9] obtained representation results for periodic ultradistributions.

In the present work we characterize the class of $\left(M_{p}\right)$ almost periodic ultradistributions in terms of classical periodicity in the sense of Bohr [3]. To this purpose we give a "second structure theorem" for the bounded ultradistributions of class $\left(M_{p}\right)$, which completes the structure results of Pilipovic [7]. Application is made to the Dirichlet Problem on the right half plane with almost periodic ultradistributional boundary values.

\section{MAIN RESUlts}

Let $D=i d / d t$; then, following Komatsu [4], an operator of the form $P(D)=$ $\sum_{n=0}^{\infty} a_{n} D^{n}$ is called ultradifferential operator of class $\left(M_{p}\right)$ if there are positive 
constants $L$ and $C$ such that

$$
\left|a_{n}\right| \leq C L^{n} M_{n}^{-1}, \quad n \in N_{0} \text {. }
$$

If the sequence $\left(M_{p}\right)$ satisfies the condition (M.2) then the following are continuous linear mappings:

$$
P(D): \mathbf{E}\left(M_{p}\right) \rightarrow \mathbf{E}\left(M_{p}\right) \quad \text { and } \quad P(D): \mathbf{D}\left(M_{p}\right) \rightarrow \mathbf{D}\left(M_{p}\right) .
$$

Lemma 1. Let $P(D)$ be a ultradifferential operator of class $\left(M_{p}\right)$; then $P(D)$ : $\mathbf{D}_{L^{1}}\left(M_{p}\right) \rightarrow \mathbf{D}_{L^{1}}\left(M_{p}\right)$ is a continuous linear mapping.

Proof. Let be $f \in D_{L^{1}}\left(M_{p}\right)$; then for every $h>0$ we have

$$
\left\|f^{(p)}\right\|_{1} \leq\|f\|_{1, h} \cdot h^{p} M_{p}, \quad p \in N_{0} .
$$

Using the inequalities (1) we obtain

$$
\begin{aligned}
\left\|\left(a_{n} D^{n} f\right)^{(p)}\right\|_{1} & \leq\left|a_{n}\right|\left\|f^{(n+p)}\right\|_{1} \leq C\|f\|_{1, h} L^{n} h^{n+p} M_{n+p} M_{n}^{-1} \\
& \leq A C\|f\|_{1, h}(H L h)^{n}(H h)^{p} M_{p}, \quad n, p \in N_{0} .
\end{aligned}
$$

Thus if $h$ is so small that $H L h<2^{-1}$, then

$$
\left\|a_{n} D^{n} f\right\|_{1, H h} \leq A C \cdot 2^{-n}\|f\|_{1, h}, \quad n \in N_{0} .
$$

Consequently, for every $0<h<(2 L)^{-1}$,

$$
\|P(D) f\|_{1, h} \leq 2 A C\|f\|_{1, h / H} .
$$

As the constant $A C$ is independent of $f$ in a bounded subset of $\mathbf{D}_{L^{1}}\left(M_{p}\right)$, we see that the convergence of the series $\sum_{n=0}^{\infty} a_{n} D^{n} f$ is absolute in the sense of Mackey. Finally the continuity of $P(D)$ is a consequence of (2).

Let $m_{p}=M_{p} / M_{p-1}, p \in N$; for $l \in R$ define

$$
P_{l}(t)=\prod_{p=1}^{\infty}\left(1+l^{2} t^{2} / m_{p}^{2}\right), \quad t \in R .
$$

As a consequence of condition (M.3) the operator

$$
P_{l}(D)=\prod_{p=1}^{\infty}\left(1+l^{2} D^{2} / m_{p}^{2}\right)
$$

is ultradifferential of class $\left(M_{p}\right)$ (see [4, p. 60]). The next result establishes the existence of a "convenient" parametrix for the ultradifferential equation $P_{l}(D) E=\delta$ and is inspired by constructions in $[2,5]$.

Lemma 2. Let $l$ and $\varepsilon$ be positive constants; then there exists two functions $\chi \in \mathbf{D}\left(M_{p}\right)$, supp $\chi \subset(-\varepsilon, \varepsilon)$, and $\Gamma \in \mathbf{D}\left(\left(M_{p}\right),[-\varepsilon, \varepsilon], H^{2} l^{-1}\right)$ such that

$$
P_{l}(D) \Gamma+\chi=\delta \text {. }
$$

Proof. Define the function $E_{l}$ on $R$ by

$$
E_{l}(t)=\frac{1}{2 \pi} \int_{-\infty}^{+\infty} P_{l}^{-1}(s) e^{i s t} d s .
$$

Since

$$
P_{l}(s)=\prod_{p=1}^{\infty}\left(1+l^{2} s^{2} / m_{p}^{2}\right) \geq 1+l^{2} s^{2} / m_{1}^{2}
$$


the function $E_{l}$ is bounded and continuous on $R$; in particular, $E_{l} \in \mathbf{D}^{\prime}\left(M_{p}\right)$. It is not difficult to see that $E_{l} \in C^{\infty}(R)$. We also note that $P_{l}(D) E_{l}=\delta$. Indeed, we have

$$
\prod_{p=1}^{\infty}\left(1+l^{2} D^{2} / m_{p}^{2}\right)\left[\int_{-k}^{k} P_{l}^{-1}(s) e^{i s t} d s\right]=\int_{-k}^{k} e^{i s t} \underset{k \rightarrow \infty}{\rightarrow} 2 \pi \delta .
$$

On the other hand, the restriction of $E_{l}$ to $R \backslash\{0\}$ can be extended analytically to $C \backslash\{0\}$ by

$$
E_{l}(z)=\frac{1}{2 \pi z} \int_{-\infty}^{+\infty} P_{l}^{-1}\left(s z^{-1}\right) e^{i s} d s
$$

Since $\left(M_{p}\right)$ is non-quasi-analytic, there exists a function $\psi_{\varepsilon} \in \mathbf{D}\left(M_{p}\right)$ such that $\psi_{\varepsilon}(t)=1$ on $(-\varepsilon / 2, \varepsilon / 2)$ and $K=\operatorname{supp} \psi_{\varepsilon} \subset(-\varepsilon, \varepsilon)$. As the real analytic functions are ultradifferentiable (see [1] or [4]) and $\mathbf{E}\left(M_{p}\right)$ is closed under the pointwise multiplication, it follows that $\left(1-\psi_{\varepsilon}\right) E_{l} \in \mathbf{E}\left(M_{p}\right)$. Applying the operator $P_{l}(D)$ to both sides of the equality $E_{l}=\psi_{\varepsilon} E_{l}+\left(1-\psi_{\varepsilon}\right) E_{l}$, we obtain

$$
P_{l}(D)\left(\psi_{\varepsilon} E_{l}\right)+P_{l}(D)\left[\left(1-\psi_{\varepsilon}\right) E_{l}\right]=\delta .
$$

Denoting now $\Gamma=\psi_{\varepsilon} E_{l}$ and $\chi=P_{l}(D)\left[\left(1-\psi_{\varepsilon}\right) E_{l}\right]$, we obtain the required equality (3).

Since $\mathbf{E}\left(M_{p}\right)$ is closed under ultradifferential operators, $\chi \in \mathbf{E}\left(\left(M_{p}\right)\right.$. In order to finish the proof we only need to show that

$$
\psi_{\varepsilon} E_{l} \in \mathbf{D}\left(\left(M_{p}\right),[-\varepsilon, \varepsilon], H^{2} l^{-1}\right) \text {. }
$$

To this purpose, for $h>0, p \in N_{0}$, and $t \in K$, where $K=[-\varepsilon, \varepsilon]$, we estimate

$$
\begin{aligned}
\frac{\left|\left(\psi_{\varepsilon} E_{l}\right)^{(p)}(t)\right|}{h^{p} M_{p}} & \leq \sum_{q=0}^{p}\left(\begin{array}{l}
p \\
q
\end{array}\right) \frac{\left|\psi_{\varepsilon}^{(p-q)}(t) \| E_{l}^{(q)}(t)\right|}{h^{p} M_{p}} \\
& \leq \frac{1}{2 \pi} \sum_{q=0}^{p}\left(\begin{array}{l}
p \\
q
\end{array}\right) \frac{\left|\psi_{\varepsilon}^{(p-q)}(t)\right|}{h^{p-q} M_{p-q}} \int_{-\infty}^{+\infty} \frac{|s|^{q}}{h^{q} M_{q}} P_{l}^{-1}(s) d s \\
& \leq \frac{1}{\pi}\left\|\psi_{\varepsilon}\right\|_{K, h / 2} \int_{-\infty}^{+\infty} e^{M\left(|s| h^{-1}\right)} P_{l}^{-1}(s) d s .
\end{aligned}
$$

Since for $\operatorname{Re} z \geq 0$ we have (see [4, p. 88]) $e^{M(|z|)} \leq\left|\prod_{p=1}^{\infty}\left(1+z / m_{p}\right)\right|$, it follows that

$$
P_{l}^{-1}(s)=\left|\prod_{p=1}^{\infty}\left(1+i l s / m_{p}\right)^{2}\right| \leq e^{-2 M(l|s|)}, \quad s \in R .
$$

On the other hand, by Lemma 3.1a) in [6] there exists $C>0$ so that

$$
e^{M(t)-M\left(H^{2} t\right)} \leq C\left(1+t^{2}\right)^{-1}, \quad t \in R .
$$

Consequently, for $h=H^{2} l^{-1}$ we obtain

$$
\begin{aligned}
\frac{\left|\left(\psi_{\varepsilon} E_{l}\right)^{(p)}(t)\right|}{h^{p} M_{p}} & \leq \frac{1}{\pi}\left\|\psi_{\varepsilon}\right\|_{K, h / 2} \int_{-\infty}^{+\infty} e^{M\left(|s| h^{-1}\right)-M(l|s|)} d s \\
& =\frac{2}{\pi}\left\|\psi_{\varepsilon}\right\|_{K, h / 2} \cdot h \int_{0}^{\infty} e^{M(t)-M\left(H^{2} t\right)} d t \leq \operatorname{const}(h)\left\|\psi_{\varepsilon}\right\|_{K, h / 2} .
\end{aligned}
$$


This yields $\Gamma \in \mathbf{D}\left(\left(M_{p},[-\varepsilon, \varepsilon], H^{2} l^{-1}\right)\right.$.

We can give now the following characterizations of the bounded ultradistributions of class $\left(M_{p}\right)$.

Theorem 1. For $T \in \mathbf{D}^{\prime}\left(M_{p}\right)$ the following statements are equivalent:

(i) $T \in \mathbf{D}_{L^{1}}^{\prime}\left(M_{p}\right)$;

(ii) there exist two functions $f, g \in C_{b}$ and a positive constant $l$ such that $T=P_{l}(D) f+g$

(iii) $T * \varphi \in C_{b}$, for every $\varphi \in \mathbf{D}\left(M_{p}\right)$; moreover if $B \subset \mathbf{D}\left(M_{p}\right)$ is bounded then the set $\{T * \varphi, \varphi \in B\}$ is uniformly bounded $C_{b}$;

(iv) for every compact $K \subset R$ there exists $h>0$ such that $T * \varphi \in C_{b}$ for every $\varphi \in \mathbf{D}\left(\left(M_{p}\right), K, h\right)$.

Proof. (ii) $\Rightarrow$ (i) The functions $f$ and $g$ are in $\mathbf{D}_{L^{1}}^{\prime}\left(M_{p}\right)$ and by Lemma 1, $\mathbf{D}_{L^{1}}^{\prime}\left(M_{p}\right)$ is closed under ultradifferentials operators of class $\left(M_{p}\right)$; hence the required statement.

(i) $\Rightarrow$ (iii) Let be $\varphi \in \mathbf{D}\left(M_{p}\right)$ and $K=\sup \varphi$; we shall prove that $T * \varphi$ is a linear continuous functional on $\mathbf{D}\left(M_{p}\right)$ endowed with the topology of $L^{1}(R)$. We denote $\check{\varphi}$ the function $\check{\varphi}(t)=\varphi(-t)$. There are positive constants $C$ and $h$ such that for every $\psi \in \mathbf{D}\left(M_{p}\right)$ we have

$$
\begin{aligned}
|\langle T * \varphi, \psi\rangle| & =|\langle T, \check{\varphi} * \psi\rangle| \leq C\|\check{\varphi} * \psi\|_{1, h}=C \sup _{p \in N_{0}} \int_{-\infty}^{+\infty} \frac{\left|\left(\check{\varphi}^{(p)}\right) \psi(t)\right|}{h^{p} M_{p}} d t \\
& \leq C \sup _{p \in N_{0}} \int_{-\infty}^{+\infty} \int_{-\infty}^{+\infty} \frac{\mid\left(\check{\varphi}^{(p)}(t-s)|| \psi(s) \mid\right.}{h^{p} M_{p}} d t d s \leq C\|\varphi\|_{1, h}\|\psi\|_{1} .
\end{aligned}
$$

Hence $T * \varphi \in C_{b}$ and

$$
\|T * \varphi\|_{\infty}=\sup _{t \in R}|(T * \varphi)(t)| \leq C\|\varphi\|_{1, h} .
$$

Further, if $B$ is a bounded subset of $\mathbf{D}\left(M_{p}\right)$ then $\sup _{\varphi \in B}\|\varphi\|_{1, k}<+\infty$, for every $k>0$, so that (5) yields $\sup _{\varphi \in B}\|T * \varphi\|_{\infty}<+\infty$.

(iii) $\Rightarrow$ (iv) Let $B_{1}$ be the unit ball in $L^{1}(R)$ and $B \subset \mathbf{D}\left(M_{p}\right)$ be bounded. Then by (iii) we have $|\langle T * \check{\varphi}, \check{\psi}\rangle|=|\langle T, \varphi * \check{\psi}\rangle|=|\langle T * \psi, \varphi\rangle|<M$ for every $\varphi \in B_{1} \cap \mathbf{D}\left(M_{p}\right)$ and $\psi \in B$ (where the constant $M$ is independent of $\varphi \in B_{1} \cap \mathbf{D}\left(M_{p}\right)$ and $\left.\psi \in B\right)$. Hence the set $\left\{T * \check{\varphi} ; \varphi \in B_{1} \cap \mathbf{D}\left(M_{p}\right)\right\}$ is bounded in $\mathbf{D}^{\prime}\left(M_{p}\right)$. Consequently, for every $K \subset R$ compact, there is $h>0$ so that

$$
|\langle T * \check{\varphi}, \check{\psi}\rangle| \leq \operatorname{const}(\psi), \quad \forall \varphi \in B_{1} \cap \mathbf{D}\left(M_{p}\right), \quad \psi \in \mathbf{D}\left(\left(M_{p}\right), K, h\right) .
$$

Hence

$$
|\langle T * \psi, \varphi\rangle| \leq \operatorname{const}(\psi)\|\varphi\|_{1}, \quad \forall \varphi \in \mathbf{D}\left(M_{p}\right), \psi \in \mathbf{D}\left(\left(M_{p}\right), K, h\right)
$$

so that $T * \psi \in C_{b}$ for every $\psi \in \mathbf{D}\left(\left(M_{p}\right), K, h\right)$.

(iv) $\Rightarrow$ (ii) Let $\varepsilon>0, K=[-\varepsilon, \varepsilon], h$ correspond to $K$ by (iv), and $\Gamma$ be the parametrix given by Lemma 2 for $l=H^{2} h^{-1}$. Then (3) yields

$$
T=T * \chi+P_{l}(D)(T * \Gamma) \text {. }
$$

By the choice of $l, \Gamma \in \mathbf{D}\left(\left(M_{p}\right),[-\varepsilon, \varepsilon], h\right)$ so that $T * \Gamma \in C_{b}$. As also $T * \chi \in C_{b}$, the representation (5) holds for $f=T * \Gamma$ and $g=T * \chi$. 
Remark. Theorem 1 is also true for the ultradistributions in the spaces $\mathbf{D}_{L^{p}}^{\prime}\left(M_{p}\right)$, as defined by Pilipovic in [7]; to this purpose we only have to replace the space $C_{b}$ by $L^{q}, q=p / p-1$, and to adapt conveniently the proof. We recall that in [7] the following weaker representation result for $T \in \mathbf{D}_{L^{p}}^{\prime}\left(M_{p}\right)$ was obtained under weaker assumptions on the sequence $\left(M_{p}\right): T=\sum_{n=0}^{\infty} f_{n}^{(n)}$ in the weak topology of $\mathbf{D}_{L^{p}}^{\prime}\left(M_{p}\right)$ where $f_{n} \in L^{q}, n \in N_{0}$, and $a_{n}=\left\|f_{n}\right\|_{q}, n \in N_{0}$, satisfy (1).

We can now give our main result.

Theorem 2. For $T \in \mathbf{D}_{L^{1}}\left(M_{p}\right)$ the following statements are equivalent:

(i) $T$ is almost periodic;

(ii) there are two function $f, g \in C_{\mathrm{ap}}$ and a positive constant $l$ such that $T=P_{l}(D) f+g$

(iii) $T * \varphi \in C_{\text {ap }}$ for every $\varphi \in \mathbf{D}\left(M_{p}\right)$;

(iv) there exists $h>0$ such that $T * \varphi \in C_{\mathrm{ap}}$ for every $\varphi \in \mathbf{D}\left(\left(M_{p}\right), K, h\right)$, $K$ compact.

Proof. (ii) $\Rightarrow$ (i) We first note that

$$
P_{l}(D)\left[e^{i \lambda t}\right]=P_{l}(\lambda) e^{i \lambda t}, \quad \lambda, t \in R .
$$

As $P_{l}(D)$ is continuous in $\mathbf{D}_{L^{1}}^{\prime}\left(M_{p}\right)$, then $P_{l}(D) T$ is a.p. whenever $T$ is a.p. On the other hand, every $f \in C_{\text {ap }}$ defines an a.p. ultradistribution of class $\left(M_{p}\right)$. Indeed, $f$ is a uniform limit of trigonometric polynomials and the uniform convergence on $R$ obviously implies the convergence in $\mathbf{D}_{L^{1}}^{\prime}\left(M_{p}\right)$. All these facts prove that under (ii) $T$ is almost periodic.

(i) $\Rightarrow$ (iii) Let $T$ be almost periodic; there exists a sequence of trigonometric polynomials $\left(P_{n}\right)$ converging to $T$ in $\mathbf{D}_{L^{1}}^{\prime}\left(M_{p}\right)$. A simple computation yields that for every $\varphi \in \mathbf{D}\left(M_{p}\right), P_{n} * \varphi$ is also a trigonometric polynomial. It is an easy matter to see that the set $\left(\tau_{s} \varphi\right)_{s \in R}$ is bounded in $\mathbf{D}_{L^{1}}\left(M_{p}\right)$, where $\left(\tau_{s} \varphi\right)(t)=\varphi(t-s)$. Then $\left(P_{n} * \varphi\right)(s)=\left\langle P_{n}, \tau_{s} \breve{\varphi}\right\rangle \rightarrow_{n}\left\langle T, \tau_{s}, \check{\varphi}\right\rangle=(T * \varphi)(s)$ and the convergence is uniform on $R$. Hence $T * \varphi \in C_{\mathrm{ap}}$.

(iii) $\Rightarrow$ (iv) Denote by $\overline{\mathbf{D}}_{L^{1}}\left(\left(M_{p}\right), h\right)$ the completion of $\mathbf{D}_{L^{1}}\left(M_{p}\right)$ in the norm $\|\cdot\|_{1, h} ;$ since by [7] $\mathbf{D}^{\prime}\left(M_{p}\right)=\bigcup_{k \rightarrow 0} \mathbf{D}_{L^{1}}^{\prime}\left(\left(M_{p}\right), k\right)$ in the set theoretical sense, there exists $k>0$ such that $T \in \mathbf{D}_{L^{1}}^{\prime}\left(\left(M_{p}\right), k\right)$. Let $h=k H^{-1}$ (with $H$ given by (M.2)); we shall show that $\mathbf{D}\left(\left(M_{p}\right), K, h\right) \subset \mathbf{D}_{L^{1}}\left(\left(M_{p}\right), k\right)$, for every $K$ compact. To this purpose consider $\psi \in \mathbf{D}\left(M_{p}\right)$ with $\int_{-\infty}^{+\infty} \psi(s) d s=1$ and $\psi_{\varepsilon}(t)=\varepsilon^{-1} \psi\left(t \varepsilon^{-1}\right)$; then for $\varphi \in \mathbf{D}\left(\left(M_{p}\right), K, h\right), \varphi_{\varepsilon}=\varphi * \psi_{\varepsilon} \in \mathbf{D}\left(M_{p}\right) \subset$ $\mathbf{D}_{L^{1}}\left(M_{p}\right)$ and $\left\|\varphi_{\varepsilon}-\varphi\right\|_{1, k} \underset{\varepsilon \rightarrow 0}{\longrightarrow} 0$. Indeed, we have

$$
\begin{aligned}
\left\|\varphi_{\varepsilon}-\varphi\right\|_{1, k} & =\sup _{p \in N_{0}} \frac{\left\|\varphi_{\varepsilon}^{(p)}-\varphi^{(p)}\right\|}{k^{p} M_{p}} \\
& =\sup _{p \in N_{0}} \frac{1}{k^{p} M_{p}} \int_{-\infty}^{+\infty} \int_{-\infty}^{+\infty}\left|\varphi^{(p)}(t+\varepsilon s)-\varphi^{(p)}(t)\right| \psi(s) d t d s \\
& \leq \varepsilon \operatorname{const}(k) \cdot \sup _{p \in N_{0}} \frac{\left\|\varphi^{(p+1)}\right\|_{1}}{k^{p} M_{p}} \\
& \leq \varepsilon A H M_{1} \operatorname{const}(k) \sup _{p \in N_{0}} \frac{H^{p}\left\|\varphi^{(p+1)}\right\|_{1}}{k^{p} M_{p+1}} \leq \varepsilon \operatorname{const}(k) \cdot\|\varphi\|_{1, h} .
\end{aligned}
$$


It is an easy matter to see now that $\tau_{s} \check{\varphi}_{\varepsilon} \rightarrow_{\varepsilon} \tau_{s} \check{\varphi}$ in the norm $\|\cdot\|_{1, k}$, uniformly for $s \in R$. Hence $\left(T * \varphi_{\varepsilon}\right)(s) \rightarrow_{\varepsilon}(T * \varphi)(s)$ uniformly on $R$. Since all the functions $T * \varphi_{\varepsilon}$ are a.p. it follows that also $T * \varphi$ is a.p.

(iv) $\Rightarrow$ (ii) We use the formula (6) as in the proof of Theorem 1 to obtain the required result.

\section{An application to the Dirichlet Problem for the half plane}

Corduneanu studied in [3] the harmonic almost periodic functions. We shall show the existence of a.p. solutions for the following Dirichlet Problem.

Proposition 1. Let $T$ be an almost periodic ultradistribution; there exists a harmonic function $u(x, y)$ in the right half-plane such that

(i) for every $x>0$, the function $y \rightarrow u(x, y)$ is a.p.;

(ii) $u(x, y) \underset{x \rightarrow 0}{\longrightarrow} T$ in $\mathbf{D}^{\prime}\left(M_{p}\right)$.

Proof. By the structure Theorem 2 we may write $T=P_{l}(D) f_{1}+f_{2}$, for some $l>0$ and $f_{1}, f_{2} \in C_{\mathrm{ap}}$. Define for $i=1,2$

$$
u_{i}(x, y)=\frac{1}{\pi} \int_{-\infty}^{+\infty} f_{i}(t) \frac{x d t}{x^{2}+(y-t)^{2}}, \quad x>0, y \in R .
$$

Then by Theorem 5.15 in [3] the functions $u_{i}, i=1,2$, are harmonic, continuous in the half plane $x \geq 0$, almost periodic for every $x>0$ and so that $\lim _{x \rightarrow 0} u_{i}(x, y)=f_{i}(y)$ uniformly with respect to $y$ on the whole axis. In particular,

$$
P_{l}(D) u_{1}(x, y)+u_{2}(x, y) \underset{x \rightarrow 0}{\longrightarrow} T \text { in } \mathbf{D}^{\prime}\left(M_{p}\right) .
$$

We shall prove now that the function $F(t)=1 /\left(1+t^{2}\right), t \in R$, is in $\mathbf{D}_{L^{1}}\left(M_{p}\right)$. Indeed from

$$
F(t)=\frac{1}{2}\left[(i t+1)^{-1}-(i t-1)^{-1}\right], \quad t \in R,
$$

we obtain for $p \in N$

$$
F^{(p)}(t)=\frac{(-i)^{p} p !}{2}\left[(i t+1)^{-(p+1)}-(i t-1)^{-(p+1)}\right] .
$$

Recall that by condition (M.3) we have for every $h>0, C_{h}>0$ such that (see [5, Lemma 2]) $p ! \leq C_{h} h^{p} M_{p}, p \in N$. Hence by means of simple computations (8) yields

$$
\left\|F^{(p)}\right\|_{1} \leq \operatorname{const}(h) h^{p} M_{p}, \quad p \in N_{0}, h>0,
$$

i.e., $F \in \mathbf{D}_{L^{1}}\left(M_{p}\right)$. It is now an easy matter to see that for $x>0$ and $y \in R$ the function $t \rightarrow x /\left(x^{2}+(y-t)^{2}\right)$ is in $\mathbf{D}_{L^{1}}\left(M_{p}\right)$. Consequently the function

$$
(x, y) \rightarrow P_{l}\left(D_{y}\right) u_{1}(x, y)=\frac{1}{\pi} \int_{-\infty}^{+\infty} f_{1}(t) P_{l}\left(D_{y}\right)\left[\frac{x}{x^{2}+(y-t)^{2}}\right] d t
$$

is harmonic $\left(P_{l}(D)\right.$ and $\Delta$ commute) in the right half plane and almost periodic for $x>0$ (the proof is similar to the case treated in [3, Theorem 5.15] but in the present case the almost periodicity is no more uniform with respect to $x>0)$. Finally, in virtue of $(7)$, the function $u(x, y)=P_{l}\left(D_{y}\right) u_{1}(x, y)+u_{2}(x, y)$ has all the required properties. 


\section{REFERENCES}

1. C. C. Chou, La transformation de Fourier complexe et l'Equation de Convolution, Lecture Notes in Math., vol. 325, Springer-Verlag, Berlin and New York, 1973.

2. I. Cioranescu and L. Zsidó, $\omega$-ultradistributions and their application to the operator theory, Banach Center Publ., vol. 8, Polish Sci. Publ., Warsaw, 1988, pp. 77-218.

3. C. Corduneanu, Almost-periodic functions, Tracts in Pure Appl. Math., vol. 22, Interscience, New York, 1968.

4. H. Komatsu, Ultradistributions. I, J. Fac. Sci. Univ. Tokyo Sect. IA Math. 20 (1973), 25105.

5. J. Körner, Roumieu'sche ultradistributionen als Randverteilung holomorpher funktionen, Dissertation, Kiel, 1975.

6. H. J. Petzsche, Die Nuklearität der Ultradistributionsräume and Satz vom Kern. I, Manuscripta Math. 74 (1978), 1-39.

7. S. Pilipović, Hilbert transformation of Beurling ultradistributions, Rend. Sem. Mat. Univ. Padova 77 (1987), 1-13.

8. L. Schwartz, Théorie des distributions, Hermann, Paris, 1973.

9. Y. Taguchi, Fourier coefficients of periodic functions of Gervey classes and ultradistributions, Yokohama Math. J. 35 (1987), 51-60.

Department of Mathematics, University of Puerto Rico, Río Piedras, Box 23355, 00931, Puerto Rico 